\title{
Research on the Relationship of Optical Illusion Deceleration Marking and Changing Rate of Pupil
}

\author{
*Ting Shang ${ }^{1}$, Boming Tang ${ }^{2}$ and Panpan $\mathrm{Tao}^{2}$ \\ ${ }^{1}$ School of Traffic \& Transportation, Chongqing Jiaotong University, \\ Chongqing, 400074, China \\ ${ }^{2}$ School of Civil Construction, Chongqing Jiaotong University, \\ Chongqing, 400074, China \\ Ting Shang,email:335304854@qq.com
}

\begin{abstract}
In order to investigate the impact of the transverse width and angle of the optical illusion deceleration marking on the change of the driver's pupil, the comb-tooth deceleration marking with the transverse width of $20-55 \mathrm{~cm}$ and angle of $0^{\circ}-180^{\circ}$ was selected for the virtual experiment in the laboratory. The changing rate of driver's pupil was used as an indicator to evaluate the safety and comfort of the width and angle of the optical illusion deceleration marking and then the quantitative relationship model for the two was established. The results showed that when the angle of the marking was $125^{\circ}$ to $150^{\circ}$, the driver's pupil size increased, and reached the peak at $150^{\circ}$; when the angle was $30^{\circ}$ to $45^{\circ}$, the change rate of the driver's pupil was smaller. When the driver got close to the optical illusion deceleration marking, the transverse width of the marking had a greater influence on the driver's pupil; and when the driver drove into the marking, the angle of the marking had a greater influence; when the driver drove away from the marking, the transverse width and angle of the marking had a coordinated influence on the driver's pupil.
\end{abstract}

Keywords : Traffic safety; Changing rate of pupil; Optical illusion deceleration marking; Widths; Angles

\section{Introduction}

Up to the end of 2013, the number of the motor vehicles in China had reached to 250 million, which posed a great threat to the road safety. There were more than 200,000 casualties caused by road traffic every year[China highway2013]. Research showed that speeding was the main cause of the road traffic. The injury risk of the traffic accident was in direct proportion to the square of the running speed, and the death risk was to the fourth power[WANG Zhengguo2000].

The current widely-used mandatory speed bumps, such as rubber deceleration ridge, spike speed bumps and shock speed humps etc. could effectively reduce the running speed, but may abrade the parts of the vehicles, affect the comfortableness of driving, cause noise pollution, and cost much more for future maintenance. Optical illusion deceleration marking could just make up for the above-mentioned defects. There were many kinds of optical illusion deceleration markings, such as comb-tooth deceleration marking, herringbone speed hump, and dragon-teeth speed hump, etc. The change of the transverse width and angle of the marking may have an illusion of a changing width of the driveway on the driver. On the basis of this optical illusion and driver's psychological natural response, the optical illusion marking was used to remind the driver to actively

*Ting Shang is the corresponding author. 
slow down before getting close to the dangerous sites so that safe driving could be assured.

Godley et al.(1999)studied about the transverse speed humps and side comb-tooth deceleration markings on the driveway with gradually narrower space. The result showed that the speed-reduction effect of the former was better than that of the latter at the early stage and the space had no impact on the speed-reduction effect.In view of the fact that the design parameters of the optical illusion deceleration markings in construction sites were determined by experience, Meyer(2001)brought up a simulated design method, i.e. the slowing effect of the deceleration markings was decided by the response of the majority of the drivers after watching the graphics. Rakha et al.'s(2006) research showed that the optical illusion deceleration markings could slow down the running speed of the vehicles. Even for the vehicles that the time headway was over 45, the markings could also reduce the speed. The slowing effect of optical illusion deceleration markings on the main roads was much better than that of on the local roads. The research conducted by Vest et al. (2005)showed that strobe lights and transverse speed humps had the best slowing effect. The latter could slow down the average speed by $2.9 \%$, and the slowing effect was even better as the vehicles accelerated the speed.The simulation experiment conducted by Castet(1993)showed that the speed of the motion section was related with the angle of that section, while the speed perception of the vertical section and sloping section were different.Sections of Belvue、Silver Lake and Meriden in the United States were delimited by optical illusion deceleration markings by Abdoulaye(2010). After five months, the slowing effect contrast experiment was carried out. Belvue had no slowing effect, and $\mathrm{V}_{85}$ increased by $0.2 \%$; Meriden slowed down by $8.2 \%$, and its $\mathrm{V}_{85}$ slowed down by $8.9 \%$, as much as Silver Lake. In Denton's(2013)report, the test section was Newbridge roundabout in Scottish Midlothian. After comparing and analyzing the data three weeks before and after the road was delimited, it showed that the slowing effect was quite good, and was slowed down by $30 \%$ and $23 \%$ at $\mathrm{V}_{85}$ and the average speed respectively.Zhu Shunying(2014)carried out the experiment from different aspects and studied the impact of the time frequency and angle of the edge marking on the speed reduction of the vehicles. By combining the simulation experiment with the actual conditions of the pavement, Yan Xianhua(2011)brought up the parameter computing method of the typical fish-bone optical illusion deceleration marking and verified the slowing effect of the marking with different parameter combinations. By comparing the speed reduction performance of the optical illusion deceleration markings with several different combinations of data through computer simulation.Zheng Fang(2008)studied about the deceleration marking of the two-lane pavement in rural areas in terms of the selection of the deceleration method and the location of the markings by classifying the outlines of the cross-section and evaluating the quantitative relations of the parameters and design parameters. The existing specification did not specify this parameter. In general, the regulations on the width of the driveway and the design of the vehicle profile size in JTG B01-2014 "highway engineering technical standards" was referred to determine the transverse width and angle of each deceleration marking in the actual study. However, it was difficult to analyze the influence on the driver exactly.

The research showed that physiological indexes could exactly reflect the influence of the road condition on the driver. Since 1970s, the scholars in America and Japan had studied the fatigue problem of the driver from psychological and physiological aspect. By measuring the speed of the vehicles, the wink frequency of the driver and the change of his heart rate etc., the document(1999)compared two S-shape pavements, which had almost the same linear parameters but had great difference in the number of traffic accidents. Elizarova(1994) made an experiment on the driver on forest road, and studied the linear structure of the road, and the influence of the running speed on the driver's blood pressure, heart rate and watching behavior and their relationship. Godley (2004)made a research on the relationship of the driveway width, driving load and 
running speed, and found that narrower driveway increased the driving load and reduced the running speed. Tal(2007)selected the mixing section and single section as the experiment section, measured and analyzed the driver's heart rate at each section.Wang Linhong(2013)studied the influence of the landscape color along the expressway on the heart rate change of the driver. Hu Jiangbi(2014) analyzed the driver's visual demand characteristics for the lighting at the entrance of the tunnel and the influence of the lighting condition on the driver's safety comfortableness and change rate of the pupils area. Therefore, by means of indoor virtual experiment using Smart Eye Pro 5.7 physiological recorder to measure the driver's physiological characteristics, this paper established the physiological quantitative indicators of driver's psychological stress so as to determine the relationship of optical illusion deceleration marking and the change of driver's pupil.

\section{Driver' Nervous Emotion and the Psychological and Physiological Change Characteristics}

With the rapid development of traffic psychology, human factors engineering and computer science, the physiological and psychological indexes of human beings were gradually applied in the field of road traffic to reflect the psychological changes of the driver.

Drivers got $80 \%$ of the information by the visual when driving.The pupil size was controlled by the sympathetic nerve and parasympathetic nerve. When the body was in a nervous and active state, the sympathetic nerve played a major role to strengthen and accelerate the heat beat, dilate the pupils and increase the capacity of the fatigue muscles. While when the body was relaxed, parasympathetic nerve played a positive role to slow down the heart beat, narrow the pupils and lower the blood pressure(LI Yong et al.2004). The effectiveness of using pupil size as an index to study the driver's stress response had been accepted by the scholars(Bot S D M, Hollander A P.2000; Chang Myungsoon et al.2001; Furuichi Tomoki et al.2002).Due to the fact that pupil size had individual differences, and the changing rate of pupil was the percentage of the increment of driver's pupil size and the pupil size in the calm state, which could reflect the driver's physiological change more objectively.Therefore,this paper chose the changing rate of driver's pupil as an indicator to evaluate the tension degree of the driver and analyzed whether the driver's pupil change was related to the transverse width and angle of the optical illusion deceleration markings and then tried to analyze its principles.

\section{Design of Test Program}

In order to avoid the potential risks of the deceleration marking to the drivers, this paper employed indoor simulation test. After the modeling and rendering with 3D MAX software, the video of several simulated straight sections was produced with a width of $3.75 \mathrm{~m}$, and total length of $500 \mathrm{~m}$. The vision height was $1.5 \mathrm{~m}$, and then comb-tooth deceleration markings of different size of width were added to a section of $300 \mathrm{~m}$.

Use Smart Eye Pro 5.7 (measurement accuracy of $0.5^{\circ}$, the sampling frequency was $60 \mathrm{~Hz}$ ) to collect the change data of the testers' pupil size when they were watching the video in the human-machine laboratory. The pupil size change data of the test driver when he passed the optical illusion deceleration markings was timely collected. 


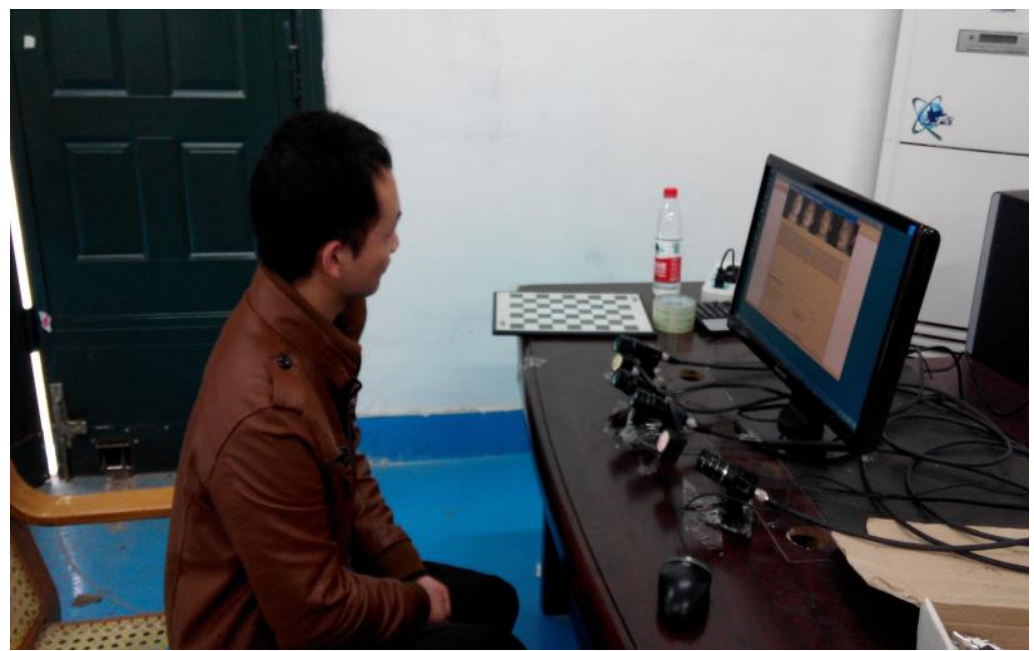

Figure 1. Experiment Site Photos

The comb-tooth optical illusion deceleration markings were symmetrically delimited along the two sides of the road, and the design parameters included the transverse width (w), the width of the marking (h), the angle $\theta$ and the distance between two markings (l) as shown in Fig. (2). According to the existing research results and the former experiment, the width of each single comb-tooth deceleration marking was $\mathrm{H}=30 \mathrm{~cm}$, and the distance between two nearby markings were $\mathrm{L}=2 \mathrm{~m}$. Besides the consideration of driver's nervous tension caused by the illusion of narrower driveway and increased running speed, how to reduce the rolling frequency, ensure safe driving in severe weather and make it convenient for construction and maintenance should also be taken into consideration in determining the width and angle of the markings.

This experiment included three stages: (1) the transverse width of the marking W was set at $20,21,22 \ldots \ldots . .55 \mathrm{~cm}$, and the angle $\theta$ was $90^{\circ}$ to test the change rate of driver's pupil caused by the illusion of narrower driveway when the angle remained unchanged; (2) the transverse width was set at $45 \mathrm{~cm}$, and the angle $\theta$ gradually increased from $5^{\circ}$ to $180^{\circ}$ in a unit of $5^{\circ}$ to determine driver's nervous emotion caused by the illusion of increasing running speed when the transverse width kept unchanged; (3) the transverse width and angle kept changed at the same time ( refer to the above two stages for the value of each parameter) to analyze the driver's pupil change influenced by these two factors.

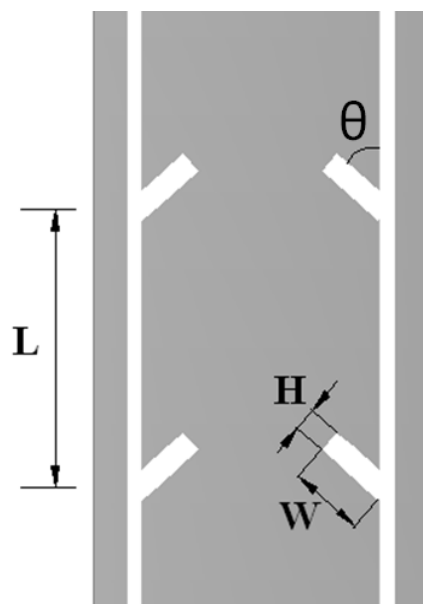

Figure 2. Geometric Parameters of Comb-tooth Optical Illusion Deceleration Marking 
Before the test, a preliminary test of the instrument was conducted to check its effectiveness. After the tester correctly wearing the Smart Eye Pro 5.7 physiological recorder, collect his resting pupil size in 5 minutes as the normal pupil size, so as to make a comparison with the dynamic pupil size during the test. The starting \& end positions and abnormal situations (such as overtaking, driveway change etc.) were recorded for easier data processing at later stages. The time recorded by the physiological recorder and manual recording should be all the same to ensure the data synchronism of all the information.

\section{Test Results Analysis}

Process the original experimental data with Smart Eye Pro 5.7 recorder and the analysis software on the basis of probability statistics theory and common sense (table 1 only listed some typical data due to the limited space), and make a further analysis of the data with Matlab software.

Table 1. Experimental Data

\begin{tabular}{cccc}
\hline No. & Marking Width/cm & Angle/ & Change Rate of pupil/\% \\
\hline 1 & 30 & 90 & 14.18 \\
2 & 31 & 90 & 22.40 \\
3 & 32 & 90 & 17.99 \\
4 & 33 & 90 & 12.79 \\
5 & 34 & 90 & 9.40 \\
6 & 35 & 90 & 21.95 \\
7 & 36 & 90 & 20.79 \\
8 & 37 & 90 & 19.41 \\
9 & 38 & 90 & 17.30 \\
10 & 39 & 90 & 22.09 \\
\hline
\end{tabular}

\subsection{The Influence of the Width W of the Marking on the Driver's Pupil Change}

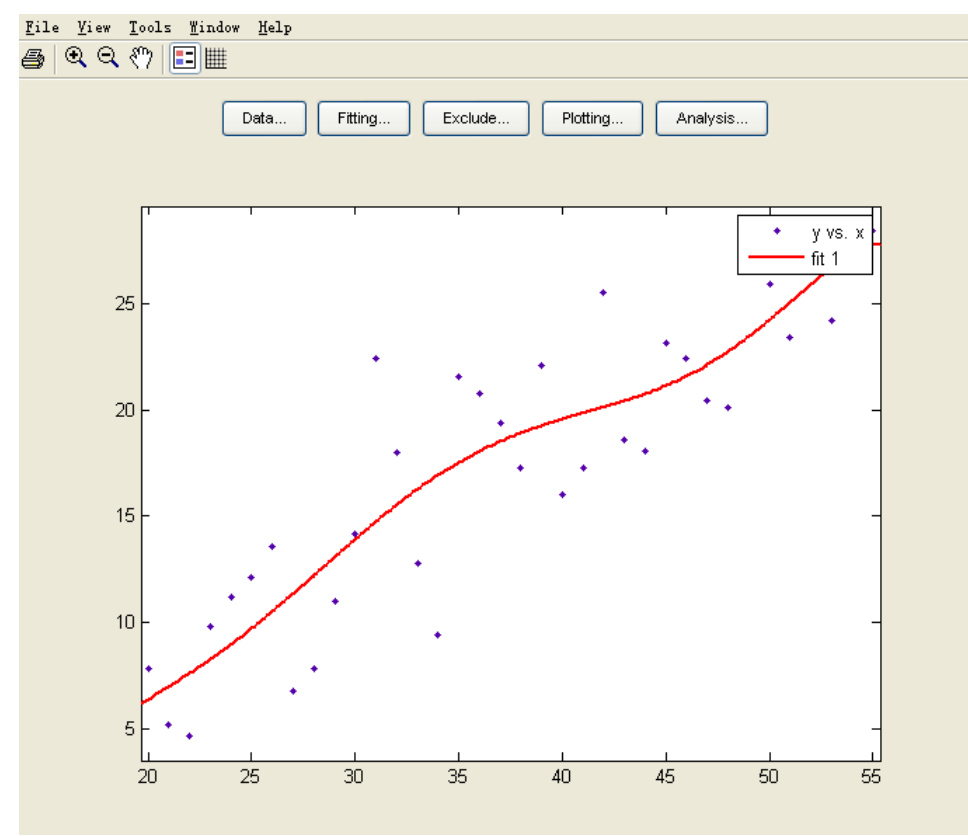

Figure 3. The Relationship of the Width of the Marking and the Changing Rate of Pupil 
After calculations, the non-linear regression model for the width of the marking and the changing rate of driver's pupil was as follows:

$$
f(x)=4.427 x^{10^{-5}} x^{x^{4}}-0.006106 x^{x^{3}}+0.296 x^{x^{2}}-5.306 x x+35.67
$$

In formula (1), $\mathrm{f}(\mathrm{x})$ was the changing rate of driver's pupil, and $\mathrm{x}$ was the width of the marking. The application range of the model was: $x \in[20,55]$. The test of goodness of fit for the regression model $\mathrm{R}^{2}$ was 0.7881 , which was very typical of the sample data and could be used to indicate the relationship of the width of the marking the change of driver's pupil.

According to Figure. 3, when the driver got close to the optical illusion deceleration marking in free flow state, he would get un-adapted for a short moment, and the changing rate of pupil would fluctuate in a small range and remain basically constant. When he drove into the deceleration markings and got stimulated by different size of width, he could not get adapted immediately and may have an illusion of narrower driveway. At this time, he would get nervous and the pupil change obviously. The illusion of narrower driveway got stronger as the increase of the width and at the same time his tension got stronger and changing rate of pupil increased. This was consistent with the theoretical basis of "changing rate of pupil increases when tension, while reduces when fatigue". When the driver drove away from the optical illusion deceleration marking, he would feel less and less nervous, and the change of pupil kept constant again.

\subsection{The Influence of the Angle $\theta$ of the Marking on the Driver's Pupil Change}

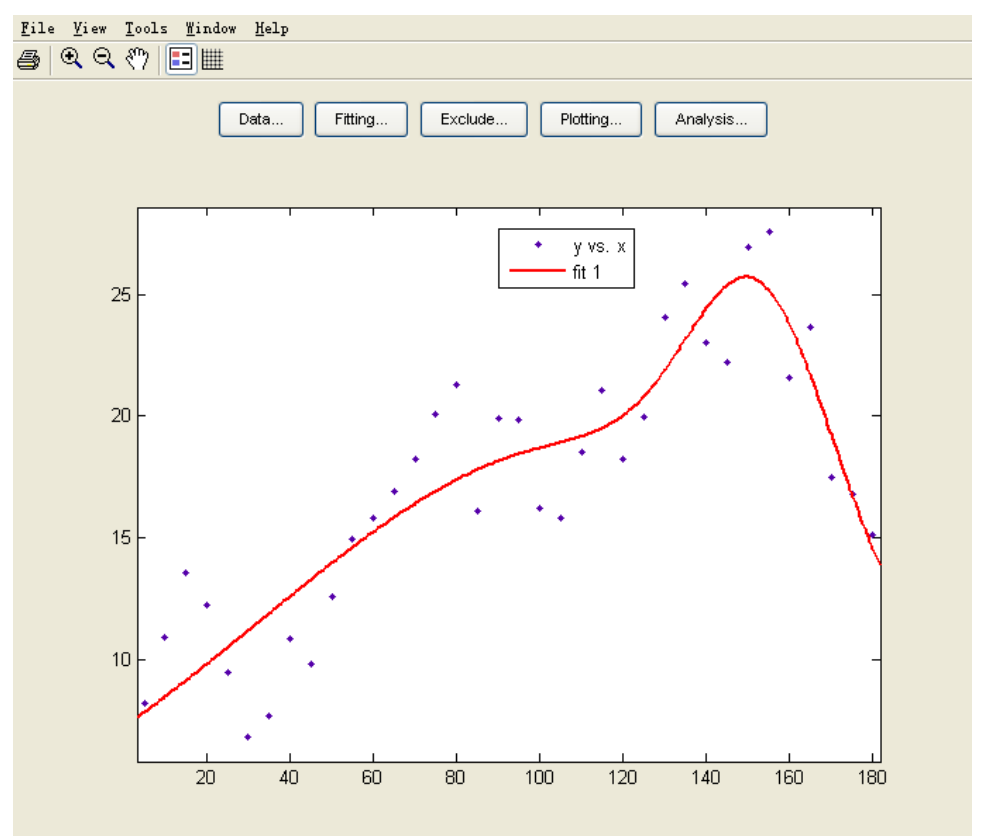

Figure 4. The Relationship of the Angle of the Marking and the Changing Rate of Pupil

After calculations, the non-linear regression model for the angle of the marking and the changing rate of driver's pupil was as follows:

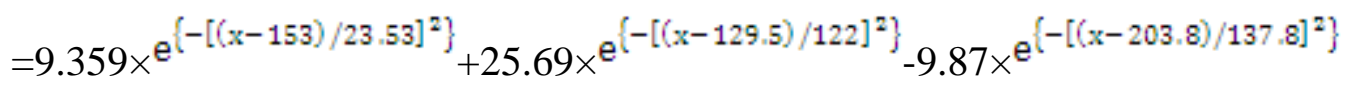

In formula (2), $\mathrm{f}(\mathrm{x})$ was the changing rate of driver' $\mathrm{s}$ pupil, and $\mathrm{x}$ was the 
angle of the marking. The test of goodness of fit for the regression model $\mathrm{R}^{2}$ was 0.8211 , which was very typical of the sample data and showed that the angle of the marking and the changing rate of the driver's pupil had a positive correlation relationship.

According to Figure 4, when the driver got close to the optical illusion deceleration marking in free flow state, his pupil fluctuated in a very small range. When he drove into the deceleration markings and got stimulated by different angle of the marking, the pupil changed differently. When the angle was $125^{\circ}$ to $150^{\circ}$, the changing rate of the pupil increased, and reached the peak at $150^{\circ}$. When the angle was $30^{\circ}$ to $45^{\circ}$, the changing rate was relatively small. The above changes were caused by Zollner illusion. When the angle was smaller than $90^{\circ}$, the dividing line of the two driveways expanded, otherwise, it shrank, and made the driver have an illusion of narrower driveway(PRIZMETAL W et al.2001). When the angle was $150^{\circ}$, the Zollner illusion was the strongest, and the pupil changed most obviously. The angle of $135^{\circ}$ took second place. When the driver drove away from the optical illusion deceleration marking, the change of pupil kept constant again.

\subsection{The Influence of Both the Width and Angle of the Marking on the Driver's Pupil Change}

3.3.1 Modeling of Trend Surface: The fitting of the trend surface was the most effective method for the space curved surface in mathematics. It approximately simulated the space change trend of the target parameter by the space curved surface in the form of function. Suppose at a certain width and angle, the pupil of the driver was $z_{i}\left(x_{i}, y_{i}\right) \quad(i=1,2, \ldots$, $\mathrm{n})$, and the fitting of the trend surface $\operatorname{was} \mathrm{f}(\mathrm{x}, \mathrm{y})$, then the residual sum of squares was as follows:

$$
\mathrm{Q}=\sum_{\mathrm{i}=1}^{\mathrm{n}}\left[\mathrm{z}_{\mathrm{i}}-f\left(x_{s} y\right)\right]_{2}
$$

When Q was the lowest, it was the fitting of the trend surface under least square. This paper used trend surface model to fit for the width and angle of the deceleration marking and driver's pupil change. Below was the fitting formula:

$\mathrm{Z}=\mathrm{a}_{0+} \sum_{\mathrm{i}=1}^{\mathrm{n}}\left[\mathrm{a}_{\mathrm{i}} \cdot \mathrm{x}^{\mathrm{i}}+\mathrm{b}_{\mathrm{i}} \cdot \mathrm{y}^{\mathrm{i}}\right]$

In the above formula, ${ }^{a_{0}} 、 a_{i} 、 b_{i}$ were all coefficients to be fitted.

3.3.2 $\mathbf{R}^{2}$ test of Trend Surface Fitting: The fitting degree coefficient of trend surface and actual surface $\mathrm{R}^{2}$ was an important indicator to test the fitness of the regression model. The percentage of the total sum of square and regression sum of square of variant $Z$ was commonly used to express the goodness of fit for regression model(KANG Zhujun2007).

$$
\begin{aligned}
\mathrm{SS}_{\mathrm{D}} & =\sum_{\mathrm{i}-1}^{\mathrm{n}}\left(\mathrm{z}_{\mathrm{i}}-\widehat{\mathrm{z}_{\mathrm{i}}}\right)^{2} \\
\mathrm{SS}_{\mathrm{R}} & =\sum_{\mathrm{i}=1}^{\mathrm{n}}\left(\mathrm{z}_{\mathrm{i}}-\overline{\mathrm{z}_{1}}\right)^{2} \\
\mathrm{SS}_{\mathrm{T}} & =\mathrm{SS}_{\mathrm{D}}+\mathrm{SS}_{\mathrm{R}}
\end{aligned}
$$

In the above formula, $\mathrm{SS}_{\mathrm{T}}$ was the sum of squares for total; $\mathrm{SS}_{\mathrm{D}}$ was residual sum of squares, which was used to show the influence of other random factors on $\mathrm{z}$ variation; $\mathrm{SS}_{\mathrm{R}}$ was regression sum of squares, which was used to indicate the total effects of all the independent variables on the dependent variables.

$$
R^{2}=\frac{S S_{R}}{S S_{T}}=1-\frac{S S_{D}}{S S_{T}}
$$


The bigger $R^{2}$ was, the higher the goodness of fit of the trend surface was.

3.3.3 Significance F Test of Fit for Trend Surface: F test of fit for trend surface was the significance testing of the total regression model of the trend surface. The exact computing method was as follows:

$$
F=\frac{S S_{R} / p}{S S_{D} / n-p-1}
$$

In the formula, $\mathrm{p}$ was the freedom degree of the regression sum of squares; $n-p-1$ was the freedom degree of the residual sum of squares. The regression equation could only be employed after passing $R^{2}$ testing and $\mathrm{F}$ testing.

\subsection{Model Calculations}

After comparing the original experimental data and quantitatively analyzing the relationship of the width and angle of the marking and the changing rate of driver's pupil, part of experimental sample data was obtained as shown in table 2 .

Table 2. Part of Experimental Sample Data

\begin{tabular}{ccc}
\hline Width $(\mathrm{cm})$ & Angle $\left(^{\circ}\right)$ & $\begin{array}{c}\text { Changing rate of pupil } \\
\text { (times/min })\end{array}$ \\
\hline 20 & 5 & 1.48 \\
21 & 10 & 2.48 \\
22 & 15 & 5.09 \\
23 & 20 & 4.08 \\
24 & 25 & 4.42 \\
25 & 30 & 4.41 \\
26 & 35 & 8.69 \\
27 & 40 & 6.08 \\
\hline
\end{tabular}

The data in table 2 showed that there was great difference in the order of magnitudes of the width and angle of the deceleration marking and the increase rate of the driver's pupil. This paper, therefore, processed the sample data with variation method. The exact computing formula was as follows:

$$
\overline{x_{i}}=\frac{x_{i}-\min \left(x_{i}\right)}{\max \left(x_{i}\right)-\operatorname{m}\left(\mathrm{n}_{i}\right)}
$$

A relation model of transverse width of the marking $\mathrm{X}$, angle of the marking $\mathrm{Y}$ and the increase rate of driver's pupil was established in accordance with the data in table 2:

$$
f=-297.2929+18.5561 X-43.0553 Z-0.1488 X^{2}-10.6707 Z^{2}
$$

$$
\text { In the formula, } Z=\frac{X}{Y} ; f \text { was a predicted value. }
$$

After calculations, the fitting coefficient of the trend surface $R^{2}$ was 0.74 , which showed that the fitting effect of the trend surface was quite good. In the significance F test, the significance level was set at 0.05 , the result obtained by calculations and table lookup was 82.648 , which was obviously higher than the critical value and showed that the fitting equation of the trend surface had passed the significance test and could be put into application. 


\subsection{Test Result Analysis}

The relation graph of the transverse width and angle of the marking and driver's pupil change was established in Matlab in accordance with the fitting formula (11) as shown in Figure. 5.

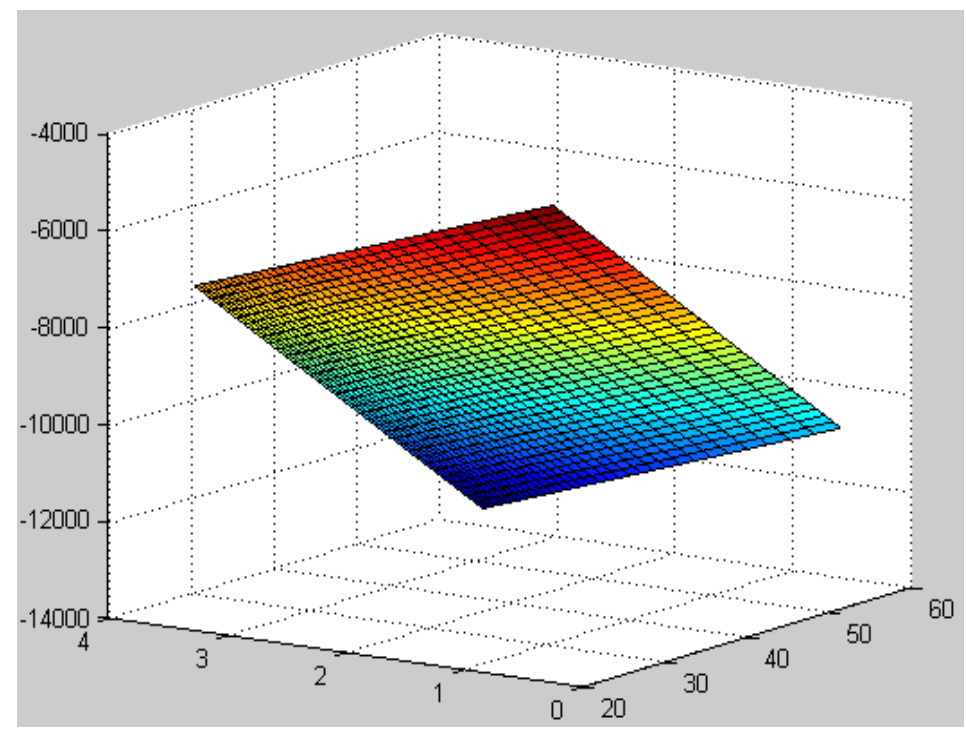

Figure 5. Relationship of the Width and Angle of the Marking and Driver's Pupil Change

According to Figure. 5, the driver's pupil increased gradually as the increase of the width of the marking, and the tension he felt was stronger than when he was calm. While as the increase of the angle, his pupil showed a tendency of an early increasing and then dropped, i.e. the change of the angle could somewhat improve driver's attention. When the influence of the width and angle of the marking existed at the same time, the driver may have an illusion of narrower driveway as the increase of the width and became more nervous when he got close to the marking; while as the angle increased from $5^{\circ}$ to $90^{\circ}$, the driver could feel the expansion of the driveway due to the impact of Zollner illusion, i.e. the driveway became broader, and the driver's tension was relieved. Of which $45^{\circ}$ was the peak. It could be concluded that under the combined influence of the width and angle, if the pupil increased slowly, the influence of the width of the marking on the driver's pupil was much greater. After the driver got into the marking, the width continued to increase; while as the angle increased from $95^{\circ}$ to $150^{\circ}$, he could feel that the driveway narrowed, i.e. the width of the driveway decreased, and the tension became stronger, of which $150^{\circ}$ was the peak. It should be noted that owing to the change of the angle, even though the transverse width was gradually increased, the length vertical to the driving direction was decreased, which had a weaker influence on the driver's pupil, however, the change rate of the pupil was still increased quickly. It showed that the angle of the marking had a more obvious influence on the change of the driver's pupil. When the driver drove away from the marking, the width continued to increase, while the length vertical to the driving direction was decreased, which could relieve the tension of the driver; when the angle increased from $155^{\circ}$ to $180^{\circ}$, the influence of the Zollner illusion was weakened, and the change rate of the driver's pupil decreased, which showed that the width and angle of the marking at this section had a coordinated influence on the driver's pupil. 


\section{Conclusions}

Based on the measurement of the parameters of the optical illusion deceleration marking and the driver's physiological and psychological indexes and the analysis of the related data from the perspectives of medical science, psychology and physiology and engineering, this paper came to the following conclusions:

(1) When the driver drove into the optical illusion deceleration marking, there was a logical relation between the changing rate of the driver's pupil and the width of the marking. The quantitative relationship model between them was thereby established.

(2) The angle of the optical illusion deceleration marking had a great influence on the driver's pupil. When the angle was $125^{\circ}$ to $150^{\circ}$, the driver's pupil increased, and reached the peak at $150^{\circ}$; when the angle was $30^{\circ}$ to $45^{\circ}$, the change rate of the driver's pupil was smaller.

(3) When the driver got close to the optical illusion deceleration marking, the transverse width of the marking had a greater influence on the driver's pupil; and when the driver drove into the marking, the angle of the marking had a greater influence; when the driver drove away from the marking, the transverse width and angle of the marking had a coordinated influence on the driver's pupil.

(4) The test analyzed the width and angle of the optical illusion deceleration marking. However, further study about the color and space of the marking were still needed. What's more, there were many kinds of optical illusion deceleration markings (such as rafter speed hump, dragon teeth speed hump, 3d stereo and speed hump, etc.), which were not included in this paper and should be taken as the research objects in the future research.

\section{References}

[1] Road Traffic Accident Casualties More than 200000 Each Year in Our Country.[EB/OL].China highway.http://www.chinahighway.com/news/2013/773652.php,2013-09-01.

[2] W Zhengguo.Traffic Injury Research of the 21st Century[J].Journal of Traumatic Surgery, 2000, vol. 2, no. 1: 2.

[3] S.A. Godley, "Driving Simulator Investigation of Perceptual Countermeasures to Speeding", Department of Psychology, Monash University, (1999).

[4] E. A. Meyer, "New Look at Optical Speed Bars[J]".In ITE Journal,vol.71, no.ll November (2001).

[5] Rakha, Hesham Ahmed; Katz, Bryan J:Duke,Dana, "Design and Evaluation of Peripheral Transverse Bars to Reduce Vehicle Speed", TRB $85^{\text {th }}$ Annual Meeting Compendium of Papers CD-ROM.Washington,DC, (2006).

[6] Vest, A. and N. Stamatiadis, "Use of Warning Signs and Markings to Reduce Speeds on Curves", TRB 84th Annual Meeting. Washington,DC, (2005).

[7] E. Castet, J. Lorenceau, M. Shiffar, "Perceived Speed of Moving Lines Depends on Orientation,Length,Speed and Luminance", Vision Research, vol. 33, no. 14, (1993), pp. 1921-1936.

[8] ABDOULAYE DIOGO BALDE.SPEED MANAGEMENT IN RURAL COMMUNITIES USING OPTICAL SPEED BARS[D].KANSAS STATE UNIVERSITY, (2010), PP. 105-109.

[9] J Ng Yew Wei, H Seng Tim, Chandrasekar S/O Palanisamy.AN ASSESSMENT ON THE EFFECTIVENESS OF TRAFFIC CALMING MARKINGS AND ALTERNATIVE FOR SPEED REGULATING STRIPS[C].16th Road Safety on Four Continents Conference,Beijing,2013:15_2.

[10] Z Shunying, Z Zi-pei., "Relationship Between Highway Edge Rate Marking Time Frequency \& Angle and Slowing Effect[J]".China Journal of Highway, 2014, vol. 3, no. 27, pp. 87-89.

[11] Y. Xianhua, "Design Research about Optical Illusion Deceleration Marking[D]".Changan University,(2011),pp. 19-20.

[12] Z Fang, "Design and Applied Research about Road Speed Facilities[D]".Jilin University,(2008), pp. 43-48.

[13] Healey, Jennifer, Seger, Justin, Picard, Rosalind.Quantifying driver stress:Developing system for collecting and processing bio-metric signals in natural situations. Biomed Sci Instrum, (1999), pp. 193-198

[14] V.V Elizarova,. E.F,Shardakova, E.G. Yamporskaya, "Electromyography evaluating neuromuscular fatigue caused by working postures of loader drivers". Gig Tr Prof Zabol, (1994), pp. 7-10.

[15] T Stuart .Godley, J Thomas.Triggs,Brian N.Fildes.Perceptuallane width,wide perceptual road centre 
markings and driving speeds[J].Ergon-omics, vol. 47, no. 3, (2004), pp. 237-256.

[16] T Oron-Gilad,A Ronen., "Road Characteristics and Driver Fatigue:A Simulator Study[J]".Traffic Injury Prevention, vol. 8, no. 3, (2007), pp. 281-289.

[17] W Lin-hong, L Shi-wu., "Impact of Roadside Landscape Color on Driver Mean Heart Rate[J]". Journal of Jilin University(Engineering and Technology Edition), vol. 43, no. 1, (2013), pp. 74-80.

[18] H Jinag-bi ,L Ran,M Yong., "Safety Threshold Evaluation Method for Expressway Tunnel Lifhting in Entrance Section[J]".China Journal of Highway and Transport, vol. 3, no. 27, (2014), pp. 95-97.

[19] LYong, YGuoen,C Yanli, "The Regulating Effect of Fatigue and Mental Load on Pupil Size in Reading[J]".Studies of Psychology and Behavior, vol. 2, no. 3,( 2004), pp. 545-548.

[20] Bot S D M, Hollander A P.The relationship between heart rate and oxygen uptake during nonsteady state exercise[J], ERGONOMICS, vol. 43, no. 10, (2000), pp. 581-592.

[21] C Myungsoon, K Juyoung, K Kyungwoo, et al. "Evaluation of driver's psycho physiological load at freeway merging area[J]".TRB, (2001), pp. 129-133.

[22] F Tomoki, J Masato Kadoma, Takeshi, "Drivers'behavior in S curve negotiations on an urban motorway[C]", //Traffic and Transport.Studies Proc. of ICTTS 2002, (2002), 2: pp. 849-856.

[23] W PRIZMETAL, D M BECK, "The Tilt-constancy Theory of Visual Illusions[J].Journal of Experimental Psychology:Human Perception and Performance, vol. 27, no. 1, (2001), pp. 206-217.

[24] K Zhu-jun.Stratum Trend Surface Fitting and DEM Modeling[D].Taiyuan:Taiyuan University of Technology, (2007), pp. 22-25.
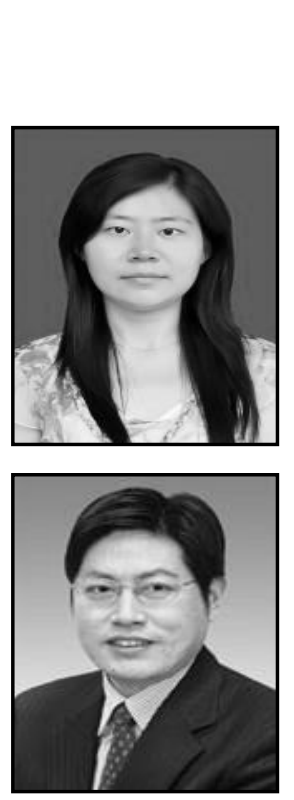

Boming Tang, He works at Chongqing Jiaotong University in China. He received B.S. degree from Southeast University in 1985, M.S. degree from Southeast University of Roads and Traffic Engineering in 1987 and Ph.D. degree from Southeast University in 1990. His research interests include Road Traffic Security, Traffic Engineering and Human Factor Engineering.

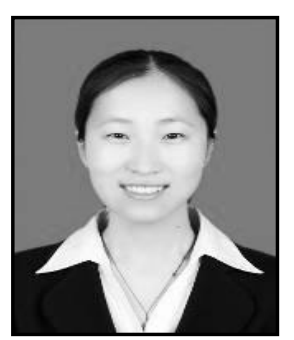

Panpan Tao, She received her B.E degree in Road Bridge and River-crossing Engineering from Shandong Agriculture University,China in 2013. Currently she is a Postgraduate student in Transportation Engineering from Chongqing Jiaotong University. Her research interests are Road Traffic Security, Traffic Psychology. 
International Journal of Security and Its Applications

Vol. 10, No. 3 (2016) 